\title{
ADAM10 mediates the cell invasion and metastasis of human esophageal squamous cell carcinoma via regulation of E-cadherin activity
}

\author{
BO MA ${ }^{1}$, HONG-YAN ZHANG ${ }^{2}$, XUE BAI $^{2}$, FENG WANG $^{2}$, XIU-HUA REN $^{3}$, \\ LEI ZHANG ${ }^{1}$ and MING-ZHI ZHANG ${ }^{1}$
}

Departments of ${ }^{1}$ Oncology and ${ }^{2}$ Pathology, The First Affiliated Hospital of Zhengzhou University, Zhengzhou, Henan 450052;
${ }^{3}$ Department of Anatomy, The Basic Medical College of Zhengzhou University, Zhengzhou, Henan 450001, P.R. China

Received December 4, 2015; Accepted January 14, 2016

DOI: 10.3892/or.2016.4667

\begin{abstract}
A disintegrin and metalloprotease 10 (ADAM10) is involved in the tumorigenesis, invasion and metastasis of several types of solid tumors. However, the potential role of ADAM10 in human esophageal squamous cell carcinoma (ESCC) is not yet well understood. The present study showed that ADAM10 was overexpressed in human ESCC tissues in vivo, and positively associated with depth of tumor invasion, lymph node metastasis and TNM stage, contributing to tumor carcinogenesis, invasion and metastasis. Additionally, ADAM10 was overexpressed in 3 types of ESCC cell lines in vitro, as compared to that in normal esophageal epithelial cells (NEECs); and moreover, ESCC cells with high ADAM10 expression obtained enhanced invasion and migration ability. Subsequently, ADAM10 silencing by small interfering (si) RNA in ESCC cell line, EC-1, reduced cell invasion, migration and proliferation in vitro. Finally, ADAM10 negatively regulated E-cadherin in ESCC in vivo and in vitro. In conclusion, active ADAM10 promotes the carcinogenesis, invasion, metastasis and proliferation of ESCC and controls invasion and metastasis at least in part through the shedding of E-cadherin activity, which makes it a potential biomarker and a useful therapeutic target for ESCC.
\end{abstract}

\section{Introduction}

Esophageal carcinoma is one of the most common malignancies and has been ranked as the sixth leading cause of cancer-related death worldwide (1-4). Esophageal squamous

Correspondence to: Professor Ming-Zhi Zhang, Department of Oncology, The First Affiliated Hospital of Zhengzhou University, 1 Jianshe East Road, Zhengzhou, Henan 450052, P.R. China E-mail: 15838385207@163.com

Key words: a disintegrin and metalloprotease 10, E-cadherin, esophageal squamous cell carcinoma, invasion, metastasis cell carcinoma (ESCC), accounting for approximately $90 \%$ of all esophageal carcinoma cases, is more predominant in Asian countries (5). In the past decades, although tremendous strides have been made in therapeutic strategies including surgical resection, radiotherapy and chemotherapy, tumor invasion and metastasis still invariably heralds a poor prognosis and a low 5-year survival rate in ESCC patients (6-8). Therefore, a better understanding of the molecular mechanisms involved in tumor invasion and metastasis and discovery of a more effective target for anticancer therapy may help to prolong the survival and improve the life quality of patients with ESCC.

The a disintegrin and metalloproteases (ADAMs), a family of zinc-dependent transmembrane proteins, contain a metalloprotease and a disintegrin-like domain (9-11) and are closely associated with the degradation of the basement membrane, cell migration, cell fusion and signal transduction $(12,13)$, thus playing important roles in the tumorigenesis, proliferation, differentiation, adhesion, invasion and metastasis of cancers (14-16). To date, approximately 40 ADAM members have been identified in this family, however only 21 are thought to function in humans (10). ADAM10 is an important proteolytically active member of this family (17). Emerging evidence suggests that ADAM10 is overexpressed in a variety of cancers and is associated with the tumor progression of breast cancer (18), non-small cell lung cancer (NSCLC) (19), nasopharyngeal carcinoma (20), oral squamous cell carcinoma $(21,22)$ tongue squamous cell carcinoma $(23)$, hepatocellular carcinoma $(24,25)$, gastric cancer (26), colon cancer (27), pancreatic carcinoma (28), bladder cancer (29) and renal cell carcinoma (30). Proteolytically active ADAM10 acts as an ectodomain-sheddase, which can release the extracellular domain of membrane-bound proteins, such as adhesion molecules, growth factors, cytokines, chemokines and receptors; through these actions they are able to disrupt the tumor microenvironment and modulate key processes involved in tumor cell proliferation, migration and angiogenesis $(31,32)$. E-cadherin is a transmembrane molecule that functions as an adhesion molecule, and is reported as an ADAM10 substrate $(33,34)$. ADAM10 activity could lead to elevated shedding of E-cadherin and loss of cell-cell contact (35), further enhancing migratory and metastatic behavior (36). 
However, to date there has been no related study concerning the role of ADAM10 in the tumorigenesis, invasion and metastasis of ESCC. Moreover, whether the potential mechanism by which ADAM10 promotes tumor progression is associated with E-cadherin shedding is still unclear.

Herein, in the present study, we first detected expression of ADAM10 protein in ESCC tissues in vivo and compared it to E-cadherin expression and clinicopathological parameters associated with tumor invasion and metastasis, including tumor differentiation, depth of invasion, lymph node metastasis and TNM stage. In addition, ADAM10 expression in ESCC cell lines was examined in vitro and compared to cell invasion and migration ability. Furthermore, we inhibited the expression of ADAM10 in ESCC cell lines by using ADAM10 siRNA and analyzed the effect of the downregulation of ADAM10 on cell proliferation, invasion and migration and the expression levels of E-cadherin in vitro.

\section{Materials and methods}

Statement of ethics. This study was performed according to ethical protocol approved by the First Affiliated Hospital of Zhengzhou University, Henan, China. All of the clinical tissue samples used in this study were collected after obtaining written informed consents. All efforts were made to minimize suffering.

Tissue specimens. A total of 122 ESCC tissue specimens and 60 adjacent normal esophageal mucosa were obatined from patients (73 male and 49 female) who underwent surgical resection at The First Affiliated Hospital of Zhengzhou University, Henan, China. None of the patients had previously received neoadjuvant radiotherapy, chemotherapy or immunotherapy. Tissue sections were cut at a thickness of $4 \mu \mathrm{m}$ for in situ hybridization (ISH) and immunohistochemistry (IHC). Clinical features of all the samples are listed in Table I. The diagnosis of ESCC was reconfirmed by two board-certified pathologists.

In situ hybridization (ISH) and evaluation of ISH staining. Paraffin-embedded slides were deparaffinized, rehydrated, and immersed in 3\% hydrogen peroxide. Then the slides were digested in pepsin solution, and incubated with pre-hybridization solution and hybridization solution sequentially. After visualization of ISH using BCIP/NBT, the slides were counterstained with Nuclear Fast Red. The digoxin-labeled probe sequences used in this study were as follows: ADAM10 forward primer, 5-GAGGAGTGTACGTGTGCCAGTT-3 and reverse primer, 5-GACCACTGAAGTGCCTACTCCA-3; E-cadherin forward primer, 5-AAAGGCCCATTTCCTAA AAACCT-3 and reverse primer, 5-TGCGTTCTCTATCCAG AGGCT-3. ADAM10- and E-cadherin-positive staining was viewed as kyano-purple granule-like material that was located in the cytoplasm. The staining index was calculated by adding the scores for the percentage of positively stained cells (1, $0-10 \% ; 2,>10-30 \% ; 3,>30-70 \%$; and $4,>70 \%$ ) and the staining intensity (1, weak; 2 , moderate; and 3 , strong). A score of $0-2$ was designated as a negative score (-), and $\geq 3$ as a positive score (+). All of the staining analyses were independently performed by two pathologists.
Immunohistochemistry (IHC) and evaluation of IHC staining. Paraffin-embedded slides were deparaffinized, rehydrated, and subjected to antigen retrieval by using a microwave oven. Then the slides were incubated with primary antibodies anti-ADAM10 (1:200, Abcam, USA) or anti-E-cadherin (1:100, Santa Cruz, CA, USA) respectively, and subsequently incubated with biotinylated anti-rabbit IgG secondary antibody. After visualization of the immunoreactivity using DAB, the slides were counterstained with hematoxylin. ADAM10and E-cadherin-positive staining was viewed as brown-yellow granule-like material that was located in the membrane and/or cytoplasm. The staining index was calculated by adding the scores for the percentage of positively stained cells $(1,0-10 \%$; $2,>10-50 \%$; and $3,>50 \%)$ and the staining intensity (1, weak; 2 , moderate; and 3, strong). A score of 0-2 was designated as a negative score $(-)$, and $\geq 3$ as a positive score $(+)$. All of the staining analyses were independently performed by two pathologists.

Cell lines and culture. Human ESCC cell line EC-1 was supplied by Professor Shihua Cao (Hong Kong University). Eca109 and TE-1 cells were maintained in the Key Laboratory of Tumor Pathology of Zhengzhou University. Normal esophageal epithelial cells (NEECs) of primary culture were obtained from fresh biopsies of esophageal mucosal tissues, during our previous experiment. All cells were cultured in RPMI-1640 medium supplemented with $10 \%$ fetal bovine serum (FBS), $100 \mu \mathrm{g} / \mathrm{ml}$ streptomycin and $100 \mathrm{U} / \mathrm{ml}$ penicillin, in a humidified incubator containing $5 \% \mathrm{CO}_{2}$ at $37^{\circ} \mathrm{C}$. Additionally, $1.0 \mathrm{ng} / \mathrm{ml}$ of epidermal growth factor (EGF) was added to the medium for the NEECs.

ADAM10 siRNA transfection of EC-1 cells. The sequences of ADAM10 siRNA and the control nonspecific siRNA were as follows: sense strand, AAAGGAUUCCCAUACUGAC and antisense strand, GUCAGUAUGGGAAUCCUUU for ADAM10; sense strand, AUCUUGAUCUUCAUUGUGC and antisense strand, GCACAAUGAAGAUCAAGAU for the nonspecific control. EC-1 cells grown until 80-90\% confluency were transfected with $100 \mathrm{nM}$ ADAM10 siRNA using Lipofectamine 2000 (Invitrogen, Carlsbad, USA) according to the manufacturer's instructions. EC-1 cells untreated or transfected with the nonspecific siRNA were used as control. The cells were cultured for an additional $48 \mathrm{~h}$ in a humidified incubator containing $5 \% \mathrm{CO}_{2}$ at $37^{\circ} \mathrm{C}$ before being harvested for further assays.

$R N A$ extraction, reverse transcription $(R T)$ and real-time $P C R$. Total RNA was extracted from the cultured cells using the TRIzol reagent (Invitrogen, CA, USA) according to the manufacturer's protocol. Two micrograms of total RNA was used for cDNA synthesis using the HiScript II RT kit (Vazyme, Nanjing, China). To evaluate ADAM10 and E-cadherin mRNA expression, real-time PCR was performed using a PRISM 7300 Sequence Detection system (Applied Biosystems, Foster City, CA, USA). $\beta$-actin was selected as an internal control. The primer sequences were as follows: forward, 5-CACGAGAAGCTGTGATTGCC-3 and reverse, 5-ATTCCGGAGAAGTCTGTGGTC-3 for ADAM10; forward, 5-TGATTCTGCTGCTCTTGCTG-3 and reverse, 
Table I. Protein and mRNA expression of ADAM10 and E-cadherin in ESCC tissues and corresponding normal esophageal mucosa.

\begin{tabular}{|c|c|c|c|c|c|c|c|c|c|}
\hline & \multirow[b]{2}{*}{$\mathrm{n}$} & \multicolumn{2}{|c|}{ ADAM10 } & \multirow[b]{2}{*}{$\chi^{2}$} & \multirow[b]{2}{*}{ P-value } & \multicolumn{2}{|c|}{ E-cadherin } & \multirow[b]{2}{*}{$\chi^{2}$} & \multirow[b]{2}{*}{ P-value } \\
\hline & & - & + & & & - & + & & \\
\hline \multicolumn{10}{|l|}{ Protein } \\
\hline Normal mucosa & 60 & 49 & 11 & 37.164 & 0.000 & 7 & 53 & 51.120 & 0.000 \\
\hline ESCC & 122 & 41 & 81 & & & 83 & 39 & & \\
\hline \multicolumn{10}{|l|}{ mRNA } \\
\hline Normal mucosa & 60 & 51 & 9 & 31.550 & 0.000 & 0 & 60 & 59.946 & 0.000 \\
\hline ESCC & 122 & 50 & 72 & & & 73 & 49 & & \\
\hline
\end{tabular}

5-CAAAGTCCTGGTCCTCTTCTCC-3 for E-cadherin; forward, 5-TTCGAGCAAGAGTGGCCA-3 and reverse, 5-AGGTAGTTTCGTGGATGCCA-3 for $\beta$-actin. ADAM10 and E-cadherin expression levels were normalized to $\beta$-actin and analyzed by using the $2^{-\Delta \Delta \mathrm{Ct}}$ method (37). All experiments were carried out in triplicate.

Protein extraction and western blot analysis. Protein was extracted from the cultured cells using RIPA lysis buffer (Well, Shanghai, China) according to the manufacturer's protocol. The protein concentration was determined using the BCA kit (Bioss, Beijing, China). For western blot analysis, $50 \mu \mathrm{g}$ of protein extract from each cell line was separated by SDS-PAGE and transferred onto a PVDF membrane. After blocking with 5\% non-fat milk in PBST for $1 \mathrm{~h}$ at room temperature, the membrane was incubated with the primary antibody (ADAM10, 1:500, $84 \mathrm{kDa}$; E-cadherin, 1:200, $120 \mathrm{kDa})$ at $4^{\circ} \mathrm{C}$ overnight. $\beta$-actin $(1: 5,000,43 \mathrm{kDa})$ was selected as a loading control. Protein bands were detected with the ECL system (Well, Shanghai, China) and exposed to X-ray film. Quantification was performed by using ImageJ analysis tool. All experiments were carried out in triplicate.

Matrigel invasion assay. Tumor cell invasion ability was analyzed using an invasion chamber (Corning ${ }^{\circledR}$, Corning, NY, USA). Briefly, a polycarbonate membrane with $8-\mu \mathrm{m}$ pores was coated with Matrigel and dried at room temperature for $1 \mathrm{~h}$ in a 24-well plate. Cells in serum-free medium were seeded into the upper chamber of the wells and RPMI-1640 with $10 \%$ FBS was added into the lower chamber as a chemoattractant. Following incubation at $37^{\circ} \mathrm{C}$ in $5 \% \mathrm{CO}_{2}$ for $24 \mathrm{~h}$, cells on the upper surface of the membrane were scrubbed away by a cotton swab. Cells invading through the membrane and adhering to the lower surface of the membrane were fixed with $95 \%$ alcohol, stained with $0.05 \%$ crystal violet for $1 \mathrm{~h}$, and quantitated by measuring the number of stained cells in five individual fields at a $\times 400$ magnification under a light microscope. All experiments were carries out in triplicate.

Scratch wound healing assay. Cell migration ability was measured by a scratch wounding healing assay. Cells were seeded into 12-well plates and incubated in RPMI-1640 with 10\% FBS medium. Following incubation for $24 \mathrm{~h}$, a scratch would was made across the center of the monolayer cells by using a sterile $200-\mu 1$ pipette tip. Subsequently, the cells were washed with PBS to remove the detached cells and incubated with serum-free medium for an additional $48 \mathrm{~h}$. Images of the cells that had migrated into the cell-free scratch wound area were acquired at a $\times 200$ magnification under a reversed light microscope. Cell migration ability was quantitated by measuring the width of the wounds in at least five representative fields and is expressed as 1 minus the average percent of the wound closure compared with the initial wound area measured. All experiments were carried out in triplicate.

Cell proliferation assay. Cell proliferation ability was measured by Cell Counting Kit-8 (CCK-8) assay. Briefly, the cells were seeded into 96 -well plates at a density of $5 \times 10^{3}$ cells/well and incubated for $0,24,48$ and $72 \mathrm{~h}$, respectively. At different time intervals, $10 \mu \mathrm{l}$ of CCK-8 solution (Sangon, Shanghai, China) was added to the corresponding wells and incubated for an additional $4 \mathrm{~h}$. The staining intensity in the medium was measured by determining the absorbance (OD values) at $450 \mathrm{~nm}$ to obtain cell growth curves. All experiments were carried out in triplicate.

Statistical analysis. All the data were analyzed using SPSS version 17.0 statistical package. The difference in counted data was examined by the $\chi^{2}$ test. Meanwhile, quantitative data are expressed as mean $\pm \mathrm{SD}$, and differences in quantitative data were examined by the t-test. A P-value $<0.05$ was defined as indicating a statistically significant result.

\section{Results}

ADAM10 expression is elevated and E-cadherin expression is reduced in ESCC tissues. Emerging evidence suggests that ADAM10 is overexpressed in a variety of cancers and participates in tumor progression (18-30). Thus, IHC was initially performed to determine the protein expression level of ADAM10 in ESCC tissues and corresponding normal esophageal mucosal tissues. ADAM10-positive signals showed brown-yellow granules in the membrane and/or cytoplasm (Fig. 1A and B). Cytoplasmic expression of ADAM10 protein was strong in the ESCC tissues (Fig. 1A), but weak in the normal esophageal mucosa (Fig. 1B). The positive rate of ADAM10 was $66.4 \%$ (81/122) in the ESCC tissues, significantly higher than that in the corresponding normal esophageal mucosa [18.3\% (11/60); $\mathrm{P}=0.000)$ (Table I). 

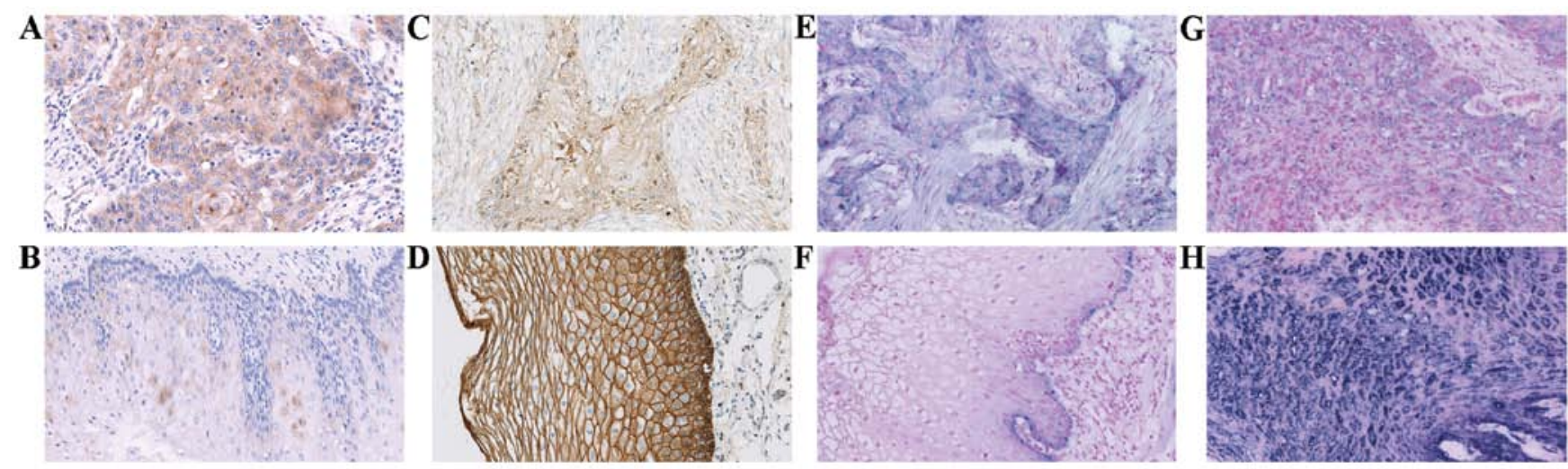

Figure 1. Immunohistochemistry (IHC) and in situ hybridization (ISH) images of ADAM10 and E-cadherin in representative samples from esophageal squamous cell carcinoma (ESCC) tissues and corresponding normal esophageal mucosa. Cytoplasmic expression of ADAM10 protein was strong in the ESCC tissues (A), but weak in the normal esophageal mucosa (B). Membranal/cytoplasmic expression of E-cadherin protein was moderate in the ESCC tissues (C), but strong in the normal esophageal mucosa (D). Cytoplasmic expression of ADAM10 mRNA was strong in the ESCC tissues (E), but was noted only in the basement of normal esophageal mucosa (F). Cytoplasmic expression of E-cadherin mRNA was weak in the ESCC tissues (G), but strong in the normal esophageal mucosa $(\mathrm{H})$. Original magnification, $\mathrm{x} 200$.

Table II. Correlation of ADAM10 and E-cadherin proteins with clinicopathological parameters in the ESCC tissues.

\begin{tabular}{|c|c|c|c|c|c|c|c|c|c|}
\hline & \multirow[b]{2}{*}{$\mathrm{n}$} & \multicolumn{2}{|c|}{ ADAM10 } & \multirow[b]{2}{*}{$\chi^{2}$} & \multirow[b]{2}{*}{ P-value } & \multicolumn{2}{|c|}{ E-cadherin } & \multirow[b]{2}{*}{$\chi^{2}$} & \multirow[b]{2}{*}{ P-value } \\
\hline & & - & + & & & - & + & & \\
\hline \multicolumn{10}{|l|}{ Gender } \\
\hline Male & 73 & 44 & 29 & 0.015 & 0.904 & 48 & 25 & 0.434 & 0.510 \\
\hline Female & 49 & 29 & 20 & & & 35 & 14 & & \\
\hline \multicolumn{10}{|l|}{ Age (years) } \\
\hline$\geq 60$ & 77 & 46 & 31 & 0.001 & 0.977 & 52 & 25 & 0.024 & 0.877 \\
\hline$<60$ & 45 & 27 & 18 & & & 31 & 14 & & \\
\hline \multicolumn{10}{|l|}{ Histological grade } \\
\hline I & 33 & 16 & 17 & 4.510 & 0.105 & 16 & 17 & 7.981 & 0.018 \\
\hline II & 51 & 14 & 37 & & & 38 & 13 & & \\
\hline III & 38 & 11 & 27 & & & 29 & 9 & & \\
\hline \multicolumn{10}{|l|}{ Depth of invasion } \\
\hline Superficial muscularis & 19 & 11 & 8 & 6.014 & 0.049 & 8 & 11 & 7.360 & 0.025 \\
\hline Deep muscularis & 46 & 14 & 32 & & & 32 & 14 & & \\
\hline Fibrous membrane & 57 & 16 & 41 & & & 43 & 14 & & \\
\hline \multicolumn{10}{|l|}{ Lymph node metastasis } \\
\hline No & 74 & 34 & 40 & 12.835 & 0.000 & 44 & 30 & 6.357 & 0.012 \\
\hline Yes & 48 & 7 & 41 & & & 39 & 9 & & \\
\hline \multicolumn{10}{|l|}{ TNM stage } \\
\hline I & 17 & 11 & 6 & 8.920 & 0.012 & 7 & 10 & 6.790 & 0.034 \\
\hline II & 44 & 14 & 30 & & & 33 & 11 & & \\
\hline III & 61 & 16 & 45 & & & 43 & 18 & & \\
\hline
\end{tabular}

Since ADAM10 mediates E-cadherin shedding and regulates epithelial cell-cell adhesion (33), we next detected the expression level of E-cadherin in the ESCC tissues and the corresponding normal esophageal mucosa tissues. E-cadherin-positive signals were presented as brown-yellow granules in the membrane and/or cytoplasm (Fig. 1C and D). Membranal and/or cytoplasmic expression of E-cadherin protein was negative or moderate (Fig. 1C) in the ESCC tissues, but strong in the normal esophageal mucosa (Fig. 1D). The positive rate of E-cadherin was $32.0 \%$ (39/122) in the ESCC tissues, significantly lower than that in the corresponding normal esophageal mucosa [88.3\% (53/60); $\mathrm{P}=0.000)$ (Table I).

Furthermore, in situ hybridization (ISH) was used to detect the mRNA expression levels of ADAM10 and E-cadherin in the ESCC tissues and corresponding normal esophageal mucosa tissues. ADAM10-and E-cadherin-positive signals 
Table III. Correlation of ADAM10 with E-cadherin protein in the ESCC tissues.

\begin{tabular}{lcccc}
\hline & \multicolumn{2}{c}{ ADAM10 } & & \\
\cline { 2 - 3 } E-cadherin & + & - & & r \\
\hline+ & 11 & 28 & -0.554 & 0.000 \\
- & 70 & 13 & & \\
\hline
\end{tabular}

both showed kyano-purple granule in cytoplasm (Fig. 1E-H). Expression of ADAM10 mRNA was strong in the ESCC tissues (Fig. 1E), but was present only in the basement of the normal esophageal mucosa (Fig. 1F). Expression of E-cadherin mRNA was negative or weak (Fig. 1G) in the ESCC tissues, but strong in the normal esophageal mucosa (Fig. 1H). The positive rates of ADAM10 and E-cadherin were $59.0(72 / 122)$ and $40.2 \%(49 / 122)$ in the ESCC tissues, and $15.0(9 / 60)$ and $100.0 \%(60 / 60)$ in the corresponding normal mucosa, respectively. Moreover, the expression of ADAM10 mRNA in the ESCC tissues was significantly higher than that in the corresponding normal esophageal mucosa $(\mathrm{P}=0.000)$, whereas contrary results were observed in the expression of E-cadherin mRNA ( $\mathrm{P}=0.000)$ (Table I).

Taken together, the above data demonstrated that overexpression of ADAM10 contributes to carcinogenesis of ESCC and this action may be attributable to ADAM10-mediated E-cadherin shedding.

Elevated ADAM10 and reduced E-cadherin protein levels are associated with tumor progression in ESCC tissues. To investigate the clinical significance of ADAM10 and E-cadherin in ESCC tissues, we analyzed the correlation of their protein expression levels with clinicopathological features including histological grade, depth of invasion, lymph node metastasis and TNM stage. As shown in Table II, ADAM10 expression was positively correlated with depth of invasion, lymph node metastasis and TNM stage $(\mathrm{P}<0.05$, respectively), but not correlated with histological grade $(\mathrm{P}>0.05)$. On the contrary, E-cadherin expression was negatively correlated with histological grade, depth of invasion, lymph node metastasis and TNM stage $(\mathrm{P}<0.05$, respectively). These data demonstrated that in the ESCC tissues elevated ADAM10 and reduced E-cadherin levels play a pivotal role in tumor progression, such as invasion and metastasis.

Negative correlation of ADAM10 and E-cadherin protein levels in ESCC tissues. To explore the role of active ADAM10 involving E-cadherin in ESCC progression, we further studied the correlation of ADAM10 protein expression with E-cadherin in the ESCC tissues. As shown in Table III, ADAM10 protein expression was significantly negatively correlated with E-cadherin ( $\mathrm{r}=-0.554, \mathrm{P}=0.000)$, implying ADAM10 may control the invasion and metastasis of ESCC through E-cadherin shedding.

ADAM10 is overexpressed in ESCC cell lines and high expression of ADAM1O is correlated with increased cell
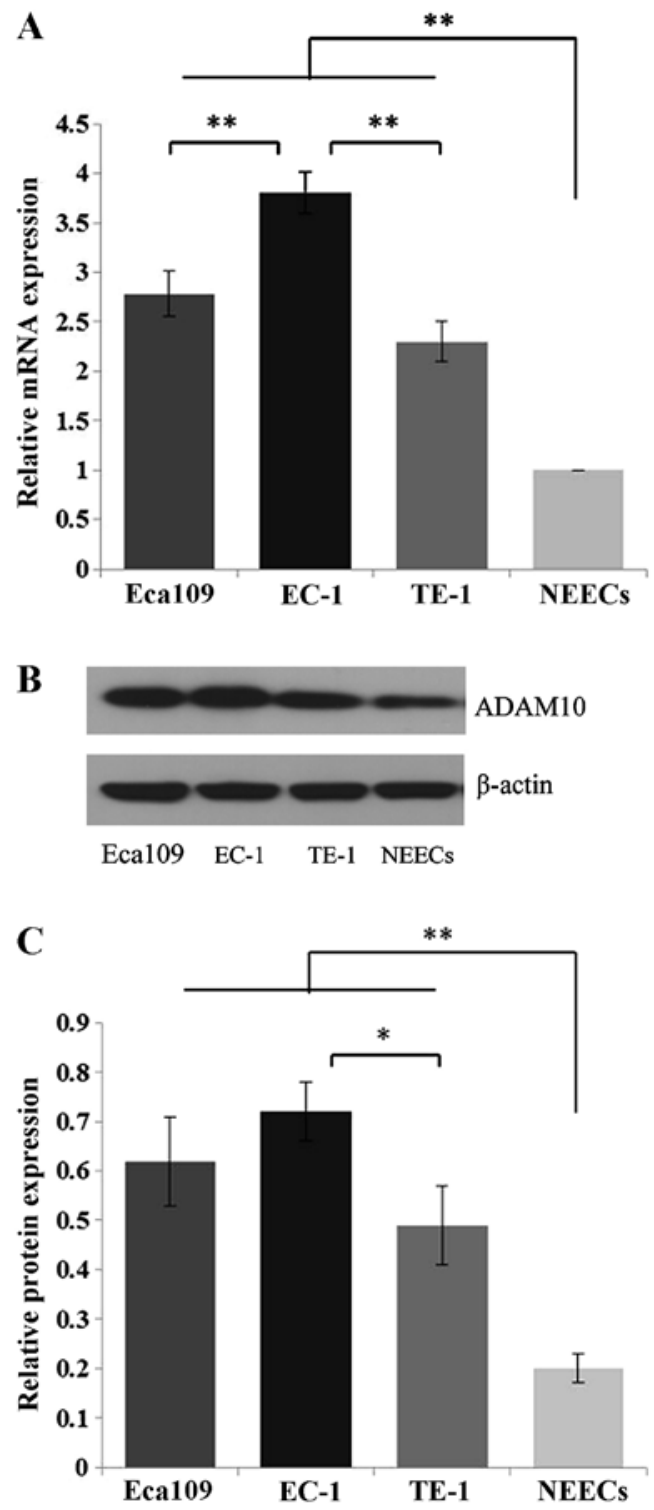

Figure 2. Quantitative real-time PCR and western blot analysis were used to detect mRNA and protein levels of ADAM10 in 3 types of ESCC cells. (A-C) Expression of ADAM10 mRNA and protein in ESCC cell lines (Eca109, EC-1, TE-1) and NEECs. ADAM10 expression at both the mRNA and protein level was significantly higher in the ESCC cells than levels in the NEECs. Moreover, this increase was more apparent in the EC-1 cells. $\beta$-actin was used as internal control. ${ }^{*} \mathrm{P}<0.05$ and ${ }^{* *} \mathrm{P}<0.01$, compared to NEECs.

invasion and migration in vitro. To further investigate the potential biological roles of ADAM10 in ESCC, cell experiments were carried out. We first used quantitative realtime PCR and western blot analysis to detect the expression levels of ADAM mRNA and protein in ESCC Eca109, EC-1 and TE- 1 cells. Compared to the NEECs, Eca109, EC-1 and TE-1 cells showed significant overexpression of ADAM10 at both the mRNA and protein levels $(\mathrm{P}<0.01)$ (Fig. 2). Moreover, this increased expression was more apparent in the EC-1 cells than that in the Eca109 and TE-1 cells at the mRNA level $(\mathrm{P}<0.01)$, and than that in the TE-1 cells at the protein level $(\mathrm{P}<0.05)$ (Fig. 2).

Based on the above clinical correlation analysis in the ESCC tissues, it is reasonable to hypothesize that ADAM10 plays an important role in promoting the invasion and migra- 
$\mathbf{A}$
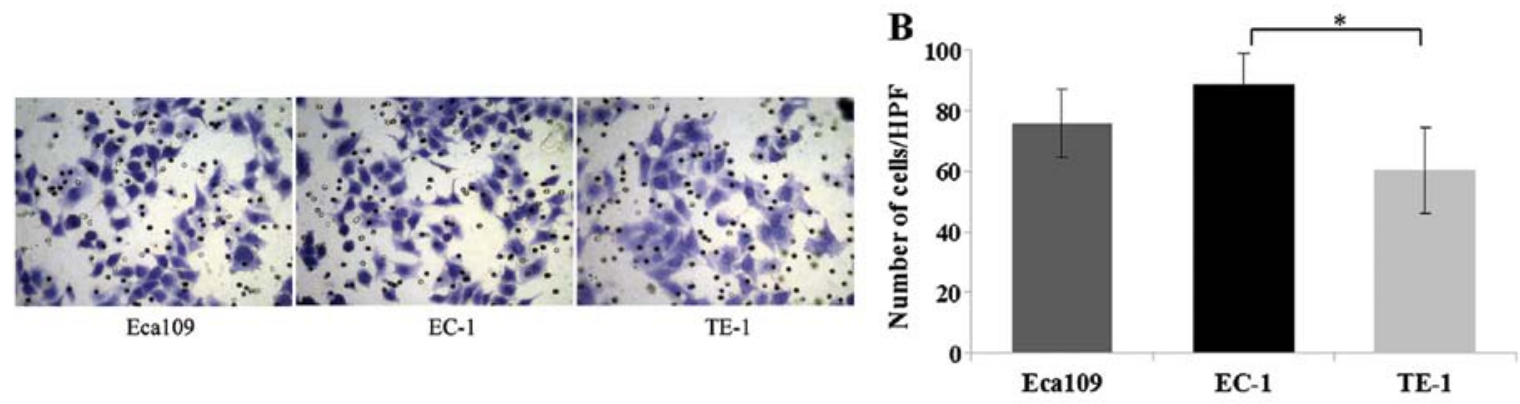

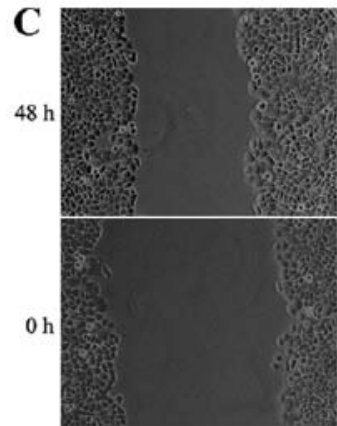

Ecal09

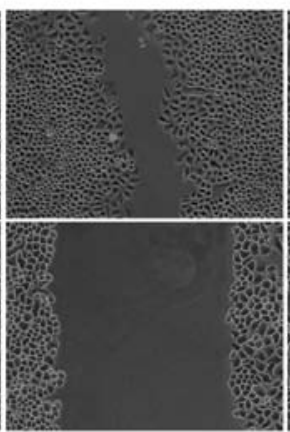

EC-1

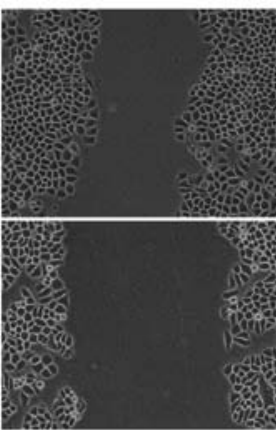

TE-1
D

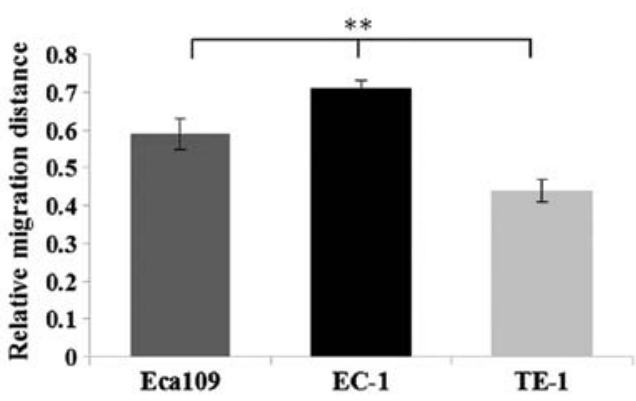

Figure 3. Matrigel invasion and scratch wound healing assays were used to detect the invasion and migration ability of ESCC cells. (A) For the Matrigel invasion assay, 3 ESCC cell lines (Eca109, EC-1, TE-1) in serum-free medium were seeded into the upper chamber and incubated for 24 h, respectively; subsequently cells invading through the Matrigel were fixed, stained and photographed under a light microscope at a $\mathrm{x} 400 \mathrm{magnification}$. (B) EC-1 cells exhibited a significant increase in the number of traversed cells as compared with the TE-1 cells. (C) For the scratch wound healing assay, 3 ESCC cell lines (Eca109, EC-1, TE-1) were seeded into 12-well plates and incubated in medium containing $10 \%$ FBS for 24 h, respectively. Subsequently the wounds were made, and the cells were incubated in serum-free medium for an additional $48 \mathrm{~h}$ and photographed under a reversed light microscope at a $200 \mathrm{magnification}$. (D) The bar graph shows that the EC-1 cell migration was more rapid than the rate noted in the TE- 1 cells. ${ }^{*} \mathrm{P}<0.05,{ }^{* *} \mathrm{P}<0.01$.

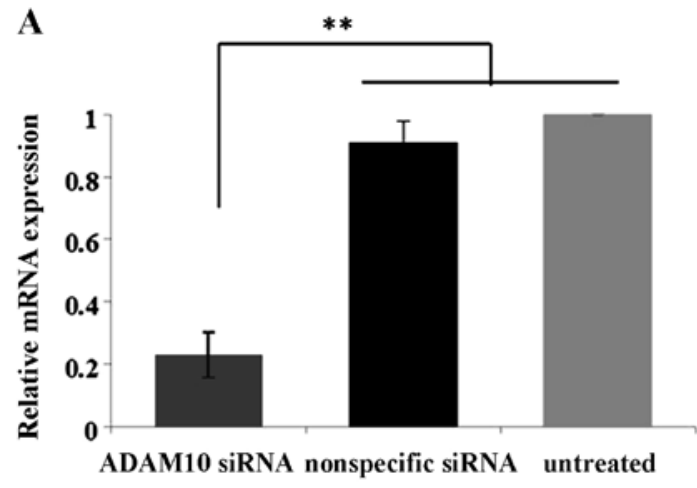

B

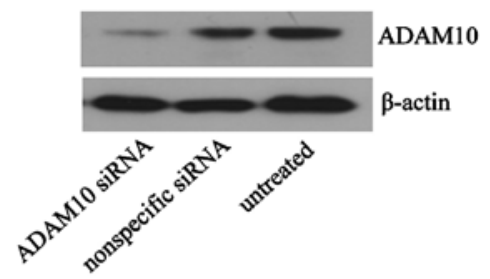

C

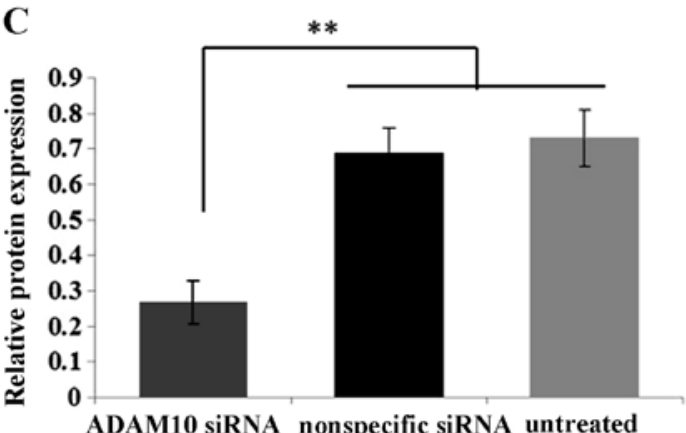

tion of ESCC cells. Therefore, we next detected the invasion and migration ability of the ESCC cells using Matrigel invasion and scratch wound healing assays, respectively. The invasion assay demonstrated that the EC-1 cells exhibited a significant increase in the number of cells that traversed the membrane toward a chemoattractant as compared with the TE-1 cells $(\mathrm{P}<0.05)$ (Fig. 3A and B). Additionally, the scratch assay demonstrated that EC-1 cells showed a significant increase in the tumor cell migration distance as compared with the Eca109 and TE-1 cells $(\mathrm{P}<0.01)$ (Fig. 3C and D).

In line with the aberrant ADAM10 in these 3 types of ESCC cell lines, EC-1 cells with high ADAM10 expression demonstrated higher invasion and migration ability, whereas TE-1 cells with low ADAM10 expression demonstrated poor invasion and migration ability, suggesting that high ADAM10 expression is consistent with increased cell invasion and migration ability.

Specific blockage of ADAM10 by ADAM10 siRNA in EC-1 cells in vitro. In order to detect the knockdown efficiency

Figure 4. Quantitative real-time PCR and western blot analysis were used to detect the specificity and efficiency of ADAM10 knockdown by ADAM10 siRNA in the EC-1 cells. (A-C) Expression of ADAM10 mRNA and protein in EC-1 cells transfected with ADAM10 siRNA, EC-1 cells transfected with nonspecific siRNA and untreated EC-1 cells. The bar graphs show that the mRNA and protein levels of ADAM10 in the EC-1 cells transfected with ADAM10 siRNA were significantly lower than those in the control nonspecific siRNA-transfected and untreated cells, indicating that the knockdown of ADAM10 by ADAM10 siRNA was specific and efficient. ${ }^{* * *} \mathrm{P}<0.01$. $\beta$-actin was used as an internal control. 
A

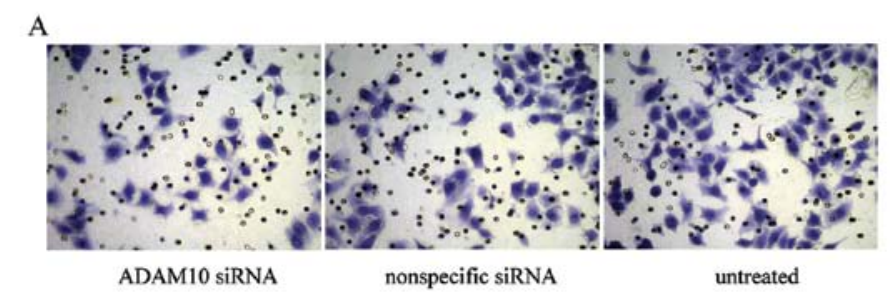

C

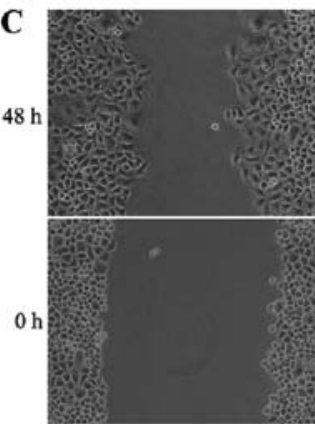

ADAM10 siRNA

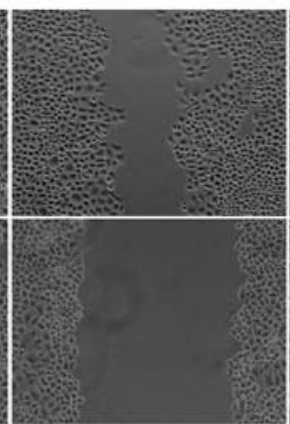

nonspecific siRNA

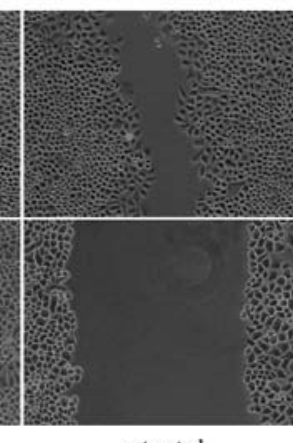

untreated

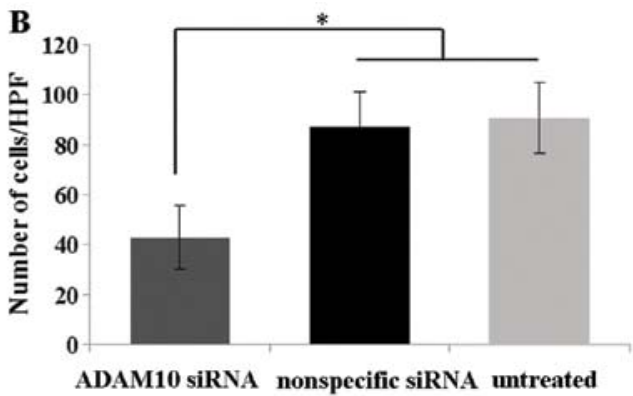

D

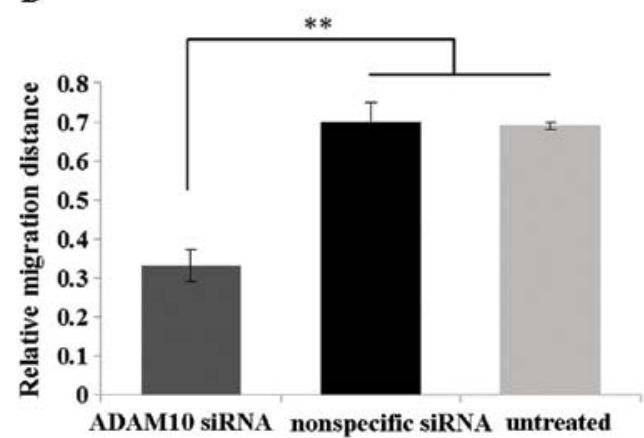

Figure 5. Matrigel invasion and scratch wound healing assays were used to detect the invasion and migration ability of the EC-1 cells transfected with ADAM10 siRNA. (A and C) Representative images of the Matrigel invasion assay (x400) and scratch wound healing assay (x200) for EC-1 cells transfected with ADAM10 siRNA, control nonspecific siRNA-transfected cells and untreated cells under a reversed light microscope, respectively. (B and D) EC-1 cells transfected with ADAM10 siRNA exhibited a significant decrease in the number of traversed cells and the cell migration distance in comparison to the control nonspecific siRNA-transfected and untreated cells, implying that ADAM10 knockdown inhibited the invasion and migration of the EC-1 cells in vitro. ${ }^{*} \mathrm{P}<0.05$, ${ }^{* *} \mathrm{P}<0.01$

of ADAM10 by ADAM10 siRNA, EC-1 cells were selected due to their elevated ADAM10 expression and enhanced invasion and migration ability compared to the Eca109 and TE-1 cell lines. Quantitative real-time PCR and western blot analysis showed that the expression levels of ADAM10 mRNA and protein in the ADAM10 siRNA-transfected cells were suppressed by $\sim 75$ and $60 \%$, significantly lower than those in the control nonspecific siRNA-transfected and untreated cells ( $\mathrm{P}<0.01)$ (Fig. 4), confirming the specific and efficient blockage of ADAM10 by ADAM10 siRNA in the EC-1 cells in vitro.

Knockdown of ADAM10 inhibits the invasion and migration of EC-1 cells in vitro. We examined the effect of ADAM10 knockdown on EC-1 cell invasion and migration using a Matrigel invasion and scratch wound healing assays. Matrigel invasion assay demonstrated that EC-1 cells transfected with the ADAM10 siRNA exhibited a significant decrease in the number of cells that traversed the membrane toward a chemoattractant as compared with the control nonspecific siRNA-transfected and untreated cells $(\mathrm{P}<0.05)$ (Fig. 5A and B). Furthermore, the scratch wound healing assay demonstrated that the EC-1 cells transfected with the ADAM10 siRNA resulted in a significant decrease in the tumor cell migration distance in comparison to the control nonspecific siRNA-transfected and untreated cells $(\mathrm{P}<0.01)$ (Fig. 5C and D). These data indicated that downregulation of ADAM10 inhibited the invasion and migration of the EC-1 cells in vitro.

Knockdown of ADAM10 inhibits proliferation of EC-1 cells in vitro. To evaluate the effect of ADAM10 knockdown

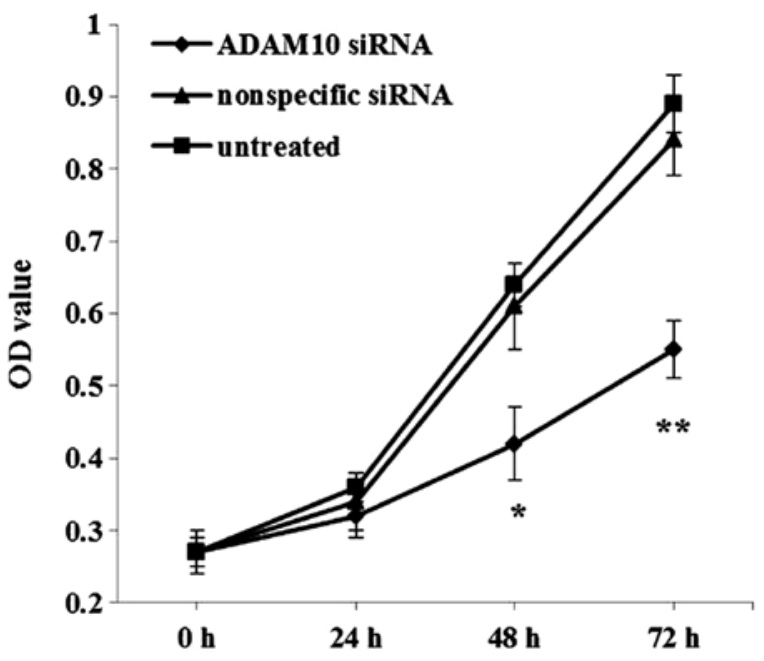

Figure 6. CCK-8 assay was used to detect the proliferation ability of the EC-1 cells transfected with ADAM10 siRNA. The EC-1 cells transfected with ADAM10 siRNA, control nonspecific siRNA-transfected cells and untreated cells were seeded into 96-well plates and incubated for 0, 24, 48 and $72 \mathrm{~h}$, respectively. At different time intervals, CCK-8 reagent was added to each well and incubated for an additional $4 \mathrm{~h}$. The staining intensity in the medium was measured by determining the absorbance (OD values) at $450 \mathrm{~nm}$ to obtain cell growth curves. Compared with the control nonspecific siRNAtransfected and untreated cells, EC-1 cells transfected with ADAM10 siRNA showed significantly decreased OD values at 48 and $72 \mathrm{~h} .{ }^{*} \mathrm{P}<0.05,{ }^{* * *} \mathrm{P}<0.01$.

on EC-1 cell proliferation, CCK-8 assay was carried out. Compared with the control nonspecific siRNA-transfected and untreated cells, the EC-1 cells transfected with the ADAM10 siRNA showed significantly decreased OD values after incubation for $48(\mathrm{P}<0.05)$ and $72 \mathrm{~h}(\mathrm{P}<0.01)$ (Fig. 6), 


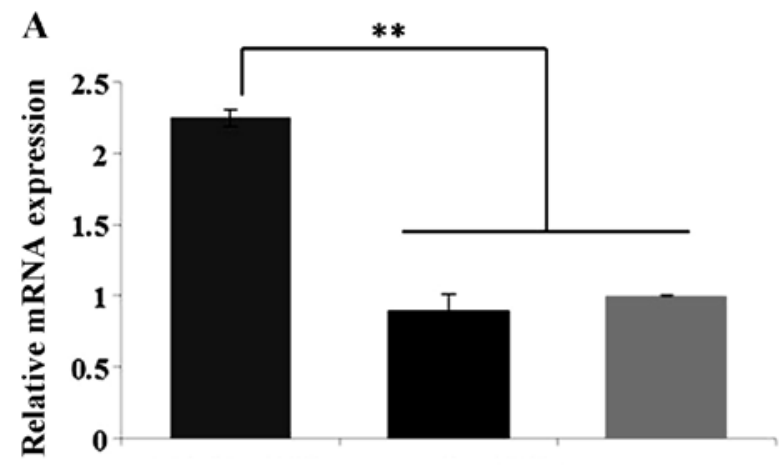

ADAM10 siRNA nonspecific siRNA untreated
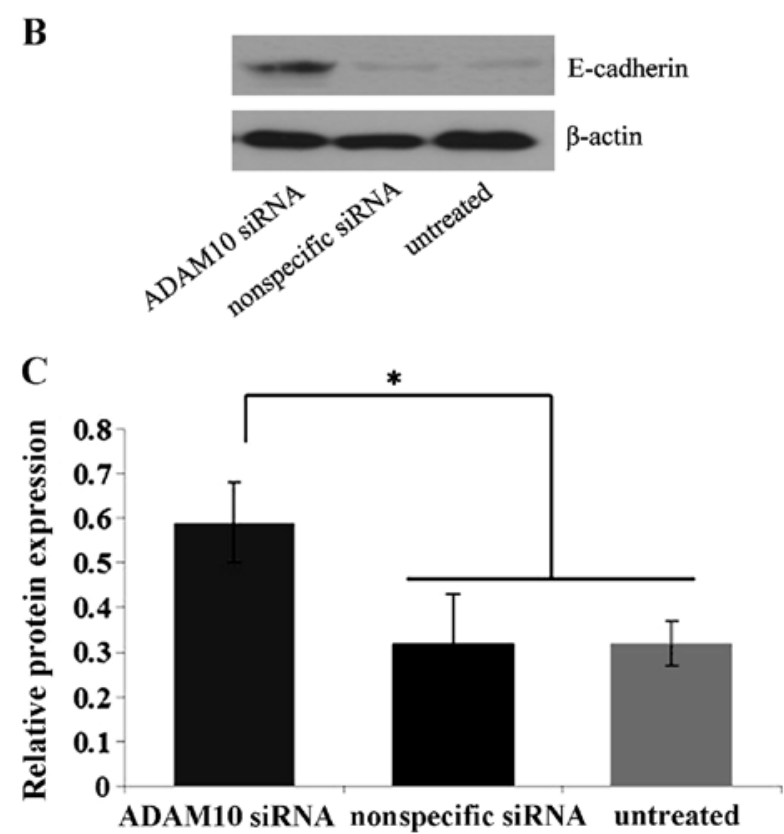

Figure 7. Quantitative real-time PCR and western blot analysis were used to detect the effect of ADAM10 knockdown by ADAM10 siRNA on E-cadherin expression in the EC-1 cells. (A-C) Expression of E-cadherin mRNA and protein in the EC-1 cells transfected with ADAM10 siRNA, EC-1 cells transfected with nonspecific siRNA and untreated EC-1 cells. The bar graphs show that the mRNA and protein levels of E-cadherin in the EC-1 cells transfected with ADAM10 siRNA were significantly higher than those in the control nonspecific siRNA-transfected and untreated cells, indicating that ADAM10 is essentially involved in E-cadherin cleavage in EC-1 cells in vitro. ${ }^{*} \mathrm{P}<0.05$ and ${ }^{* *} \mathrm{P}<0.01$. $\beta$-actin was used as an internal control.

suggesting that downregulation of ADAM10 inhibited EC-1 cell growth in vitro.

Knockdown of ADAM10 suppresses E-cadherin expression in EC-1 cells in vitro. As described previously, expression levels of ADAM10 and E-cadherin were inversely correlated in the ESCC tissues. To further characterize the intrinsic relationship between ADAM10 and E-cadherin expression and explore the possible impact that ADAM10 exerts on the invasion and metastasis of ESCC, the expression changes in E-cadherin mRNA and protein in the EC-1 cells transfected with ADAM10 siRNA were determined by quantitative real-time PCR and western blot analysis assays. As shown in Fig. 7, downregulation of ADAM10 by ADAM10 siRNA dramatically increased E-cadherin mRNA and protein in the ADAM10 siRNA-transfected EC-1 cells as compared with the control nonspecific siRNA-transfected and untreated cells $(\mathrm{P}<0.05)$. These results demonstrated that ADAM10 is essentially involved in E-cadherin cleavage in EC-1 cells in vitro.

\section{Discussion}

The role of ADAM10 has been clarified in a variety of important biological processes, such as cell adhesion (33), cell migration (38), immunoreaction regulation $(39,40)$ and apoptosis control (41). However recently, it has emerged as a major and key player in human diseases. ADAM10 is considered to contribute to Alzheimer's disease (42) and chronic inflammatory diseases (43) such as lung inflammation (44) and eczematous dermatitis (45); most importantly, it is overexpressed in many cancers and participates in tumor progression (18-30). Guo et al (19) demonstrated that ADAM10 protein expression is significantly increased in human NSCLC tissues, particularly in metastatic tissues; furthermore, downregulation of ADAM10 by ADAM10 shRNA reduced the invasion and migration of NSCLC cells. Consistent with this result, Gaida et al (28) also found that ADAM10 mRNA and protein were overexpressed in pancreatic carcinoma compared to normal pancreatic tissues, and ADAM10 silencing markedly reduced the invasiveness and migration of the tumor cells. However, the role of ADAM10 in ESCC is not yet well understood.

In the present study, we first examined the expression levels of ADAM10 mRNA and protein in human ESCC tissues and cells. As expected, ADAM10 mRNA and protein were both dramatically elevated in the ESCC tissues and cells in comparison to these levels in the normal esophageal epithelial mucosa tissues and cells, suggesting that aberrant activation of ADAM10 plays a fundamental role in the carcinogenesis of ESCC. Subsequently, the correlation of ADAM10 with tumor invasion and metastasis was analyzed. In ESCC tissues, ADAM10 expression was positively correlated with depth of invasion, lymph node metastasis and high TNM stage of the cancers; in addition, in ESCC cells, EC-1 with high ADAM10 expression exhibited higher invasion and migration ability, whereas TE-1 with low ADAM10 expression exhibited poor invasion and migration ability. Since EC-1 cells possessed a higher level of ADAM10 expression and markedly enhanced invasion and migration ability compared to the Eca109 and TE-1 cells, EC-1 cells were chosen to perform follow-up experiments to ascertain the effect of ADAM10 silencing on cell invasion and migration. After the knockdown of ADAM10 using ADAM10 siRNA, the EC-1 cells exhibited a significant decrease in the number of invasive cells, cell migration distance and cell proliferation in comparison to the control nonspecific siRNA-transfected and untreated cells. All these above results demonstrated that aberrant ADAM10 is associated with tumor cell invasion, migration, metastasis and proliferation in ESCC in vitro and in vivo.

A large body of evidence has shown that tumor invasion, metastasis and proliferation are associated with the shedding activity of ADAM10. Nevertheless, the precise mechanism ADAM10 exerts in the course of ESCC progression is still poorly understood. ADAM10 promotes tumor progression through the cleavage of its substrates, such as adhesion molecules (22). E-cadherin, an adhesion molecule, has been known 
as an ADAM10 substrate (33). ADAM10 has been implicated in the shedding of E-cadherin in response to Helicobacter (H.) pylori infection in gastric cancer cell lines (46). H. pylori infection was found to correlate with increased ADAM10 expression in gastric cancer patient samples and to induce ADAM10 expression in gastric cancer cell lines (47). Indeed, chemical inhibition or shRNA-mediated knockdown of ADAM10 resulted in decreased soluble E-cadherin generation in response to $H$. pylori infection, suggesting that induction of ADAM10 by $H$. pylori in gastric cancer promotes E-cadherin cleavage (46). In the present study, we analyzed the correlation of ADAM10 with E-cadherin in ESCC tissues and found a significantly negative correlation between them, suggesting that ADAM10 promotes the progression of ESCC via E-cadherin shedding. Accordingly, we next detected the effect of ADAM10 silencing on the shedding of E-cadherin in EC-1 cells in vitro. The data showed that the knockdown of ADAM10 dramatically promoted E-cadherin mRNA and protein in the ADAM10 siRNA-transfected cells as compared with the control nonspecific siRNA-transfected and untreated cells, indicating that ADAM10 is critically involved in the proteolytic processing of E-cadherin in EC-1 cells in vitro and ADAM10-mediated cleavage of E-cadherin represents a potent mechanism for regulating invasion, migration, and proliferation of EC-1 cells. ADAM10 could shed the extracellular domain of E-cadherin, and allow $\beta$-catenin to translocate to the nucleus (33). Nuclear translocation of $\beta$-catenin further contributes to the activation and transcription of genes involved in cell proliferation such as c-myc and cyclin D1 (48). In addition to enhanced cell proliferation, E-cadherin shedding was found to simultaneously weaken cadherin-mediated cell-cell adhesion and promote dissociation of potentially invasive and metastatic cells located in primary cancers; moreover, such dissociation could directly place malignant cells on their pathway to metastasis (10).

In conclusion, the present study suggests that ADAM10 is a key regulator in the invasion and metastasis of ESCC, and may control invasion and metastasis at least in part through the regulation of E-cadherin activity. Disruption of ADAM10 expression may provide a possible route for therapeutic intervention for ESCC.

\section{Acknowledgements}

This project was financially supported by the Education Program of Henan Province, China (grant no. 12A310014).

\section{References}

1. Ferlay J, Shin HR, Bray F, Forman D, Mathers C and Parkin DM: Estimates of worldwide burden of cancer in 2008: GLOBOCAN 2008. Int J Cancer 127: 2893-2917, 2010.

2. Zhu YH, Fu L, Chen L, Qin YR, Liu H, Xie F, Zeng T, Dong SS, Li J, Li Y, et al: Downregulation of the novel tumor suppressor DIRAS1 predicts poor prognosis in esophageal squamous cell carcinoma. Cancer Res 73: 2298-2309, 2013.

3. Parkin DM, Bray F, Ferlay $J$ and Pisani P: Global cancer statistics, 2002. CA Cancer J Clin 55: 74-108, 2005.

4. Parkin DM, Bray FI and Devesa SS: Cancer burden in the year 2000. The global picture. Eur J Cancer 37 (Suppl 8): S4-S66, 2001.

5. Tsai ST, Wang PJ, Liou NJ, Lin PS, Chen CH and Chang WC: ICAM1 is a potential cancer stem cell marker of esophageal squamous cell carcinoma. PLoS One 10: e0142834, 2015.
6. Shimada H, Shiratori T, Okazumi S, Matsubara H, Nabeya Y, Shuto K, Akutsu Y, Hayashi H, Isono K and Ochiai T: Have surgical outcomes of pathologic T4 esophageal squamous cell carcinoma really improved? Analysis of 268 cases during 45 years of experience. J Am Coll Surg 206: 48-56, 2008.

7. Radisky ES and Radisky DC: Matrix metalloproteinase-induced epithelial-mesenchymal transition in breast cancer. J Mammary Gland Biol Neoplasia 15: 201-212, 2010.

8. Pisani P, Parkin DM, Bray F and Ferlay J: Estimates of the worldwide mortality from 25 cancers in 1990 . Int J Cancer 83: 18-29, 1999.

9. Primakoff $P$ and Myles DG: The ADAM gene family: surface proteins with adhesion and protease activity. Trends Genet 16: 83-87, 2000.

10. Duffy MJ, Mullooly M, ODonovan N, Sukor S, Crown J, Pierce A and McGowan PM: The ADAMs family of proteases: new biomarkers and therapeutic targets for cancer? Clin Proteomics 8: 9, 2011.

11. Wolfsberg TG, Primakoff P, Myles DG and White JM: ADAM, a novel family of membrane proteins containing a disintegrin and metalloprotease domain: multipotential functions in cell-cell and cell-matrix interactions. J Cell Biol 131: 275-278, 1995.

12. Walker JL, Fournier AK and Assoian RK: Regulation of growth factor signaling and cell cycle progression by cell adhesion and adhesion-dependent changes in cellular tension. Cytokine Growth Factor Rev 16: 395-405, 2005.

13. Mazzocca A, Coppari R, De Franco R, Cho JY, Libermann TA, Pinzani $\mathrm{M}$ and Toker A: A secreted form of ADAM9 promotes carcinoma invasion through tumor-stromal interactions. Cancer Res 65: 4728-4738, 2005.

14. Mochizuki S and Okada Y: ADAMs in cancer cell proliferation and progression. Cancer Sci 98: 621-628, 2007.

15. Klein T and Bischoff R: Active metalloproteases of the a disintegrin and metalloprotease (ADAM) family: biological function and structure. J Proteome Res 10: 17-33, 2011.

16. Lu X, Lu D, Scully M and Kakkar V: ADAM proteins - therapeutic potential in cancer. Curr Cancer Drug Targets 8: 720-732, 2008.

17. Hartmann D, de Strooper B, Serneels L, Craessaerts K, Herreman A, Annaert W, Umans L, Lübke T, Lena Illert A, von Figura K, et al: The disintegrin/metalloprotease ADAM 10 is essential for Notch signalling but not for alpha-secretase activity in fibroblasts. Hum Mol Genet 11: 2615-2624, 2002.

18. Lendeckel U, Kohl J, Arndt M, Carl-McGrath S, Donat H and Röcken C: Increased expression of ADAM family members in human breast cancer and breast cancer cell lines. J Cancer Res Clin Oncol 131: 41-48, 2005.

19. Guo J, He L, Yuan P, Wang P, Lu Y, Tong F, Wang Y, Yin Y, Tian J and Sun J: ADAM10 overexpression in human non-small cell lung cancer correlates with cell migration and invasion through the activation of the Notch1 signaling pathway. Oncol Rep 28: 1709-1718, 2012.

20. You B, Shan Y, Shi S, Li X and You Y: Effects of ADAM10 upregulation on progression, migration, and prognosis of nasopharyngeal carcinoma. Cancer Sci 106: 1506-1514, 2015.

21. Jones AV, Lambert DW, Speight PM and Whawell SA: ADAM 10 is over expressed in oral squamous cell carcinoma and contributes to invasive behaviour through a functional association with $\alpha v \beta 6$ integrin. FEBS Lett 587: 3529-3534, 2013.

22. Ko SY, Lin SC, Wong YK, Liu CJ, Chang KW and Liu TY: Increase of disintergin metalloprotease 10 (ADAM10) expression in oral squamous cell carcinoma. Cancer Lett 245: 33-43, 2007.

23. Shao Y, Sha XY, Bai YX, Quan F and Wu SL: Effect of a disintegrin and metalloproteinase 10 gene silencing on the proliferation, invasion and migration of the human tongue squamous cell carcinoma cell line TCA8113. Mol Med Rep 11: 212-218, 2015.

24. Yue Y, Shao Y, Luo Q, Shi L and Wang Z: Downregulation of ADAM10 expression inhibits metastasis and invasiveness of human hepatocellular carcinoma HepG2 cells. BioMed Res Int 2013: 434561, 2013.

25. Zhang W, Liu S, Liu K, Wang Y, Ji B, Zhang X and Liu Y: A disintegrin and metalloprotease (ADAM) 10 is highly expressed in hepatocellular carcinoma and is associated with tumour progression. J Int Med Res 42: 611-618, 2014.

26. Wang YY, Ye ZY, Li L, Zhao ZS, Shao QS and Tao HQ: ADAM 10 is associated with gastric cancer progression and prognosis of patients. J Surg Oncol 103: 116-123, 2011. 
27. Gavert N, Sheffer M, Raveh S, Spaderna S, Shtutman M, Brabletz T, Barany F, Paty P, Notterman D, Domany E, et al: Expression of L1-CAM and ADAM10 in human colon cancer cells induces metastasis. Cancer Res 67: 7703-7712, 2007.

28. Gaida MM, Haag N, Günther F, Tschaharganeh DF, Schirmacher P, Friess H, Giese NA, Schmidt J and Wente MN Expression of a disintegrin and metalloprotease 10 in pancreatic carcinoma. Int J Mol Med 26: 281-288, 2010.

29. Fu L, Liu N, Han Y, Xie C, Li Q and Wang E: ADAM10 regulates proliferation, invasion, and chemoresistance of bladder cancer cells. Tumour Biol 35: 9263-9268, 2014.

30. Doberstein K, Pfeilschifter J and Gutwein P: The transcription factor PAX2 regulates ADAM10 expression in renal cell carcinoma. Carcinogenesis 32: 1713-1723, 2011.

31. Turner SL, Blair-Zajdel ME and Bunning RA: ADAMs and ADAMTSs in cancer. Br J Biomed Sci 66: 117-128, 2009.

32. Woods N, Trevino J, Coppola D, Chellappan S, Yang S and Padmanabhan J: Fendiline inhibits proliferation and invasion of pancreatic cancer cells by interfering with ADAM10 activation and $\beta$-catenin signaling. Oncotarget 6: 35931-35948, 2015.

33. Maretzky T, Reiss K, Ludwig A, Buchholz J, Scholz F, Proksch E, de Strooper B, Hartmann D and Saftig P: ADAM10 mediates E-cadherin shedding and regulates epithelial cell-cell adhesion, migration, and beta-catenin translocation. Proc Natl Acad Sci USA 102: 9182-9187, 2005.

34. Dittmer A, Hohlfeld K, Lützkendorf J, Müller LP and Dittmer J: Human mesenchymal stem cells induce E-cadherin degradation in breast carcinoma spheroids by activating ADAM10. Cell Mol Life Sci 66: 3053-3065, 2009.

35. Lee SB, Schramme A, Doberstein K, Dummer R, Abdel-Bakky MS, Keller S, Altevogt P, Oh ST, Reichrath J, Oxmann D, et al: ADAM10 is upregulated in melanoma metastasis compared with primary melanoma. J Invest Dermatol 130: 763-773, 2010.

36. Bánkfalvi A, Krassort M, Buchwalow IB, Végh A, Felszeghy E and Piffkó J: Gains and losses of adhesion molecules (CD44 E-cadherin, and beta-catenin) during oral carcinogenesis and tumour progression. J Pathol 198: 343-351, 2002.

37. Livak KJ and Schmittgen TD: Analysis of relative gene expression data using real-time quantitative PCR and the 2(-Delta Delta C(T)) Method. Methods 25: 402-408, 2001.

38. Mancia F and Shapiro L: ADAM and Eph: how Ephrin-signaling cells become detached. Cell 123: 185-187, 2005.
39. Hasegawa-Ishii S, Shimada A, Inaba M, Li M, Shi M, Kawamura N, Takei S, Chiba Y, Hosokawa M and Ikehara S: Selective localization of bone marrow-derived ramified cells in the brain adjacent to the attachments of choroid plexus. Brain Behav Immun 29: 82-97, 2013.

40. Gibb DR, Saleem SJ, Chaimowitz NS, Mathews J and Conrad DH: The emergence of ADAM10 as a regulator of lymphocyte development and autoimmunity. Mol Immunol 48: 1319-1327, 2011.

41. Schulte M, Reiss K, Lettau M, Maretzky T, Ludwig A, Hartmann D, de Strooper B, Janssen O and Saftig P: ADAM10 regulates FasL cell surface expression and modulates FasL-induced cytotoxicity and activation-induced cell death. Cell Death Differ 14: 1040-1049, 2007.

42. Endres K and Fahrenholz F: The role of the anti-amyloidogenic secretase ADAM10 in shedding the APP-like proteins. Curr Alzheimer Res 9: 157-164, 2012.

43. Crawford HC, Dempsey PJ, Brown G, Adam L and Moss ML: ADAM10 as a therapeutic target for cancer and inflammation. Curr Pharm Des 15: 2288-2299, 2009.

44. Dreymueller D, Uhlig S and Ludwig A: ADAM-family metalloproteinases in lung inflammation: potential therapeutic targets. Am J Physiol Lung Cell Mol Physiol 308: L325-L343, 2015.

45. Maretzky T, Scholz F, Köten B, Proksch E, Saftig P and Reiss K: ADAM10-mediated E-cadherin release is regulated by proinflammatory cytokines and modulates keratinocyte cohesion in eczematous dermatitis. J Invest Dermatol 128: 1737-1746, 2008.

46. Schirrmeister W, Gnad T, Wex T, Higashiyama S, Wolke C, Naumann $\mathrm{M}$ and Lendeckel $\mathrm{U}$ : Ectodomain shedding of E-cadherin and c-Met is induced by Helicobacter pylori infection. Exp Cell Res 315: 3500-3508, 2009.

47. Yoshimura T, Tomita T, Dixon MF, Axon AT, Robinson PA and Crabtree JE: ADAMs (a disintegrin and metalloproteinase) messenger RNA expression in Helicobacter pylori-infected, normal, and neoplastic gastric mucosa. J Infect Dis 185: 332-340, 2002.

48. Balzer EM and Konstantopoulos K: Intercellular adhesion: mechanisms for growth and metastasis of epithelial cancers. Wiley Interdiscip Rev Syst Biol Med 4: 171-181, 2012. 